\title{
Caracterização de argilas encontradas em solos de Terras Indígenas do RS com 0 auxílio da espectroscopia Mössbauer de ${ }^{57} \mathrm{Fe}$
}

\section{(Characterization of clays found in soils of the Indian Territories in Rio Grande do Sul State by using the ${ }^{57} \mathrm{Fe}$ Mössbauer spectroscopy)}

\author{
C. A. S. Pérez ${ }^{1}$, D. Gobbi ${ }^{1}$, J. L. N. Marcos ${ }^{1}$, C. Paduani ${ }^{2}$, J. D. Ardisson ${ }^{3}$ \\ ${ }^{1}$ Instituto de Ciências Exatas e Geociências, Universidade de Passo Fundo, Campus I Bairro S. José, \\ BR 285, C. P. 611, Passo Fundo, RS, 99001-970 \\ ${ }^{2}$ Departamento de Física, Universidade Federal de Santa Catarina \\ Florianópolis, SC, 88040-900 \\ ${ }^{3}$ Centro de Desenvolvimento da Tecnologia Nuclear (CDTN) \\ Belo Horizonte, $M G$, 30123-970
}

\begin{abstract}
Resumo
As argilas encontradas em amostras de solos, provenientes de Terras Indígenas do planalto médio do Rio Grande do Sul, foram caracterizadas com o intuito de fornecer dados e parâmetros técnicos para seu uso como matéria prima de produtos cerâmicos. O estudo mineralógico efetuado nas amostras utilizando a técnica de difração de raios X demonstrou que o argilomineral predominante é a caulinita. Outros minerais, como quartzo e rutilo, encontram-se presentes em menor quantidade. As análises químicas demonstram que as quantidades de óxido de $\mathrm{Mg}, \mathrm{Mn}, \mathrm{K}, \mathrm{Na}, \mathrm{Ca}, \mathrm{Cu}$ e $\mathrm{Zn}$ são baixas (a soma das porcentagens é menor que $0,97 \%$ ). As amostras foram analisadas por espectroscopia Mössbauer de ${ }^{57} \mathrm{Fe}$. Os espectros Mössbauer obtidos na temperatura ambiente confirmam a presença do mineral caulinita. Os espectros a $77 \mathrm{~K}$ revelam a existência dos minerais goetita e hematita, na forma de partículas magnéticas ultrafinas (nanopartículas) em estado superparamagnético. Os testes físicos efetuados nas amostras indicam que os solos são finos e apresentam características granulométricas e de plasticidade, que podem ser aproveitadas para a produção de materiais cerâmicos para construção ou objetos ornamentais.
\end{abstract}

Palavras-chave: matéria-prima, argilas, efeito Mössbauer.

\begin{abstract}
Clay samples collected from soils of indian territories of the middle plateau in Rio Grande do Sul were analyzed with the aim to obtain characterization data and technical parameters for their potential use as raw material for ceramic products. The mineralogical study in samples by using the X-ray diffraction technique demonstrated that the predominant clay mineral is kaolinite. Others minerals as quartz and rutile also are present in small amounts. Chemical analysis shows low percentages for oxides of $\mathrm{Mg}, \mathrm{Mn}, \mathrm{K}, \mathrm{Na}, \mathrm{Ca}, \mathrm{Cu}$ and $\mathrm{Zn}$ (overall percentages smaller than 0.97\%). The samples also were analyzed with the ${ }^{57} \mathrm{Fe}$ Mössbauer spectroscopy. The Mössbauer spectra at room temperature confirm the presence of the mineral kaolinite. At $77 \mathrm{~K}$ they reveal the existence of the minerals goethite and hematite as ultrafine magnetic particles in a superparamagnetic state. The physical tests performed in the samples show that these soils are very fine material and present appropriate granulometric characteristics and plasticity, which can be taken in advantage for the production of materials for construction or production of ornamental artifacts.
\end{abstract}

Keywords: raw clayey materials, clays, Mössbauer effect.

\section{INTRODUÇÃO}

A necessidade do homem trabalhar com solos encontra sua origem nos tempos mais remotos, podendo-se mesmo afirmar ser esta tão antiga quanto a civilização. Os solos são materiais que resultam do intemperismo ou meteorização das rochas, por desintegração mecânica ou decomposição química. Os seus componentes básicos são os minerais (primários e secundários), a matéria orgânica, ar e água.

Os minerais encontrados nos solos são elementos ou compostos químicos, via de regra, resultantes de processos inorgânicos, com composição química definida, arranjo 
atômico ordenado e encontrados naturalmente na crosta terrestre, sendo em geral sólidos. Os principais grupos de minerais secundários existentes nos solos são os argilominerais e os oxihidróxidos (óxidos e hidróxidos)

Os argilominerais pertencem à classe mineral dos filossilicatos, são constituídos pela superposição de lâminas tetraédricas de silício e lâminas octaédricas de alumínio, e ocorrem predominantemente na argila (fração com diâmetro $<2 \mu \mathrm{m}$ ) encontrada nos solos. Os argilominerais podem ser agrupados conforme a sua composição química e a seqüência do agrupamento das lâminas tetraédricas e octaédricas. Os argilominerais mais importantes encontrados nos solos são os do tipo 1:1 e 2:1. Os argilominerais 1:1 (grupo da caulinita) são formados pela superposição contínua de uma lâmina tetraédrica seguida de uma lâmina octaédrica [1].

Dentre os minerais 2:1 temos o grupo das esmectitas (montmorilonitas) e das micas. A estrutura destes minerais consiste de uma superposição contínua de duas lâminas tetraédricas para cada lâmina octaédrica. As esmectitas são expansivas e apresentam substituição isomórfica: o $\mathrm{Al}$ nas lâminas octaédricas pode ser substituído por $\mathrm{Mg}$ e Fe, entre outros cátions. O Al pode, por sua vez, substituir o Si nas lâminas tetraédricas. As micas são minerais não expansivos, apresentam átomos de $\mathrm{K}$ entre as lâminas tetraédricas e substituição isomórfica parcial no tetraedro de Si [1].

A argila encontrada nos solos é matéria-prima utilizada na fabricação de uma grande série de produtos cerâmicos. As razões para isto são: a plasticidade, a resistência mecânica após a queima, possibilita a aplicação de técnicas de processamento simples, e é disponível em grandes quantidades. Normalmente é possível fabricar produtos de cerâmica vermelha com argilas variadas, encontradas praticamente em quase todo lugar do mundo, as quais permitem utilizar diversas técnicas de processamento [2]. Entretanto, é de se esperar produtos com propriedades bem diversificadas devido às particularidades da matéria-prima utilizada. Em geral, a matéria-prima, é uma mistura complexa de diferentes variedades mineralógicas, que se apresentam como partículas de tamanho, forma e propriedades físicas e químicas diferentes. Conclui-se então que, a composição química e mineralógica dos solos, a sua distribuição granulométrica e as suas características de plasticidade são fatores que determinam a sua possível utilização como matéria-prima para a produção de produtos de cerâmica. Por isso, é de extrema importância realizar provas experimentais sobre os mesmos.

Diversas são as técnicas experimentais utilizadas para a caracterização mineralógica dos solos. Entre as mais difundidas, destacam-se a difração de raios $\mathrm{X}$ e as técnicas de análises térmicas (termogravimetria e análise térmica diferencial).

A difração de raios $\mathrm{X}$ é a técnica que por si só oferece informações mais amplas, precisas ou detalhadas quanto à caracterização e quantificação dos minerais presentes nos solos. Mesmo assim, em algumas situações, a devida identificação de algumas espécies minerais (principalmente óxidos e hidróxidos) por meio dos difratogramas é prejudicada pela superposição, baixa intensidade e/ou alargamento das linhas de difração. Isto, em geral, ocorre devido à pouca concentração e ao baixo grau de cristalização do mineral [3].

A espectroscopia Mössbauer de ${ }^{57} \mathrm{Fe}$ é uma técnica analítica de grande importância no estudo de compostos que contém Fe. Esta espectroscopia é baseada no fenômeno de absorção ou emissão ressonante nuclear de raios gama sem perda de energia por recuo. Devido à grande resolução desta técnica, é possível determinar o estado de valência dos átomos de $\mathrm{Fe}$ e o tipo de geometria dos sítios ocupados. Medidas quantitativas de parâmetros como o deslocamento isomérico (IS), o desdobramento quadrupolar elétrico (EQ) e o campo magnético hiperfino (BHF) podem ser utilizadas para distinguir entre os diferentes estados de oxidação do $\mathrm{Fe}$ em vários sítios estruturais de um composto, e detectar óxidos de Fe de diferentes tamanhos de partículas. Também é possível determinar as quantidades relativas de cada estado ou fase estrutural [4].

A presença de átomos de Fe é muito comum em compostos naturais. No caso dos solos podem ser encontrados na forma de óxidos e hidróxidos, ou como cátions substitutos do $\mathrm{Al}$ nas lâminas octaédricas e do Si nas lâminas tetraédricas dos diversos argilominerais. Assim, a análise de amostras de solos por espectroscopia Mössbauer pode confirmar e acrescentar detalhes ao estudo mineralógico efetuado pela difração de raios X [5].

$O$ presente trabalho visa estudar, utilizando técnicas experimentais laboratoriais, as propriedades mineralógicas e físico-mecânicas de amostras de solos provenientes de Terras Indígenas localizadas no planalto médio do RS. Esta pesquisa tem o intuito de fornecer dados técnico-científicos sobre as argilas encontradas nas amostras que permitam avaliar a possibilidade de seu uso como matéria-prima cerâmica.

\section{MATERIAIS E MÉTODOS}

Os estudos do presente trabalho foram realizados utilizando-se um total de seis amostras, coletadas no horizonte superficial (profundidade entre 40 e $60 \mathrm{~cm}$ ) de solos situados em três Terras Indígenas localizadas na região do planalto médio do Estado do Rio Grande do Sul (veja a Tabela I).

Tabela I - Dados geográficos e características das amostras estudadas (S1A, S2A, S2B, S3A, S3B e S3C).

[Table I-Geographic data and characteristics of the studied samples ( $S 2 A, S 2 B, S 3 A, S 3 B$ and $S 3 C$ ).]

\begin{tabular}{cccc}
\hline $\begin{array}{c}\text { Terra } \\
\text { Indígena }\end{array}$ & $\begin{array}{c}\text { Município de } \\
\text { localização }\end{array}$ & Amostra & Cor \\
\hline Carreteiro & Água Santa, RS & S1A & Marrom \\
\hline Guarita & Redentora, RS & S2A & Marrom \\
\cline { 3 - 4 } & & S2B & Cinza \\
\hline Inhacora & São Valério do Sul, RS & S3A & Cinza \\
\cline { 3 - 4 } & & S3B & Marrom \\
\cline { 3 - 4 } & & S3C & Preto \\
\hline
\end{tabular}


A fração de cada uma das amostras com diâmetro inferior a $0,075 \mathrm{~mm}$ (material passante através da peneira $\mathrm{N}^{\circ} 200$ : argila, silte e areia fina) foi caracterizada sob diversos aspectos: composição química, difração de raios $\mathrm{X}$ e espectroscopia Mössbauer de ${ }^{57} \mathrm{Fe}$. Foram determinados os limites de Atterberg e a distribuição granulométrica das amostras in natura.

Para análise química as amostras foram submetidas à digestão ácida para sua abertura. A concentração de $\mathrm{SiO}_{2}$ foi determinada por diferença de peso, e, as porcentagens de óxidos de $\mathrm{Al}$, Fe, $\mathrm{Mn}, \mathrm{Mg}, \mathrm{Na}, \mathrm{K}, \mathrm{Cu}$ e $\mathrm{Zn}$ foram determinadas por espectrofotometria de absorção atômica.

A caracterização por difração de raios $\mathrm{X}$ foi efetuada em amostras não orientadas em um difratômetro Philips PW1830/ 25, utilizando-se a radiação $\mathrm{Cu}-\mathrm{k}_{\alpha}$.

Os espectros Mössbauer das amostras em pó foram obtidos à temperatura ambiente na geometria de transmissão no modo de aceleração constante, usando um espectrômetro convencional de 512 canais com uma fonte de ${ }^{57} \mathrm{Co}$ difundida em uma matriz de Rh. A calibração foi efetuada usando-se uma folha de ferro metálico $(\alpha-\mathrm{Fe})$. Os valores dos desvios isoméricos (IS) dos espectros são dados relativos ao centro do espectro de calibração. Também foram efetuadas medidas de efeito Mössbauer à temperatura do nitrogênio líquido, $77 \mathrm{~K}$.

As características de plasticidade: limite de plasticidade (LP), limite de liquidez (LL) e o índice de plasticidade (IP); foram determinadas pelo ensaio de Casagrande, segundo as normas brasileiras NBR6459-84 e 7080-84. A análise granulométrica das amostras foi efetuada de acordo com a norma brasileira 7181-84. Até o tamanho de 200 mesh, as amostras foram classificadas por peneiramento. Abaixo deste diâmetro, a técnica utilizada foi a de sedimentação.

\section{RESULTADOS E DISCUSSÃO}

Os resultados da análise química quantitativa de óxidos presentes nas amostras são apresentados na Tabela II. Desta Tabela é possível inferir que a composição de óxido de silício, $\mathrm{SiO}_{2}$, varia pouco entre as amostras de solos: de 54,23\% até $63,33 \%$. Os teores de $\mathrm{Al}_{2} \mathrm{O}_{3}$, componente que confere refratariedade aos materiais cerâmicos, variam de 8,59 a $19,12 \%$. Observa-se, também, que a quantidade de óxido de ferro, $\mathrm{Fe}_{2} \mathrm{O}_{3}$, varia de $4,50 \%$ até $11,50 \%$. As concentrações de
$\mathrm{K}_{2} \mathrm{O}$ e $\mathrm{Na}_{2} \mathrm{O}$, componentes que atuam como fundentes nos solos, são pequenas. As baixas concentrações de $\mathrm{MgO}$ e $\mathrm{CaO}$, sugerem que os minerais que formam as amostras não são do tipo carbonato.

A análise dos difratogramas de raios $\mathrm{X}$ das amostras (Fig. 1) sugere a existência de minerais de argila e quartzo. Da presença de quartzo, resultam picos bem definidos e de grande intensidade na maioria dos difratogramas. Este fato dificulta a devida caracterização e identificação de outras fases

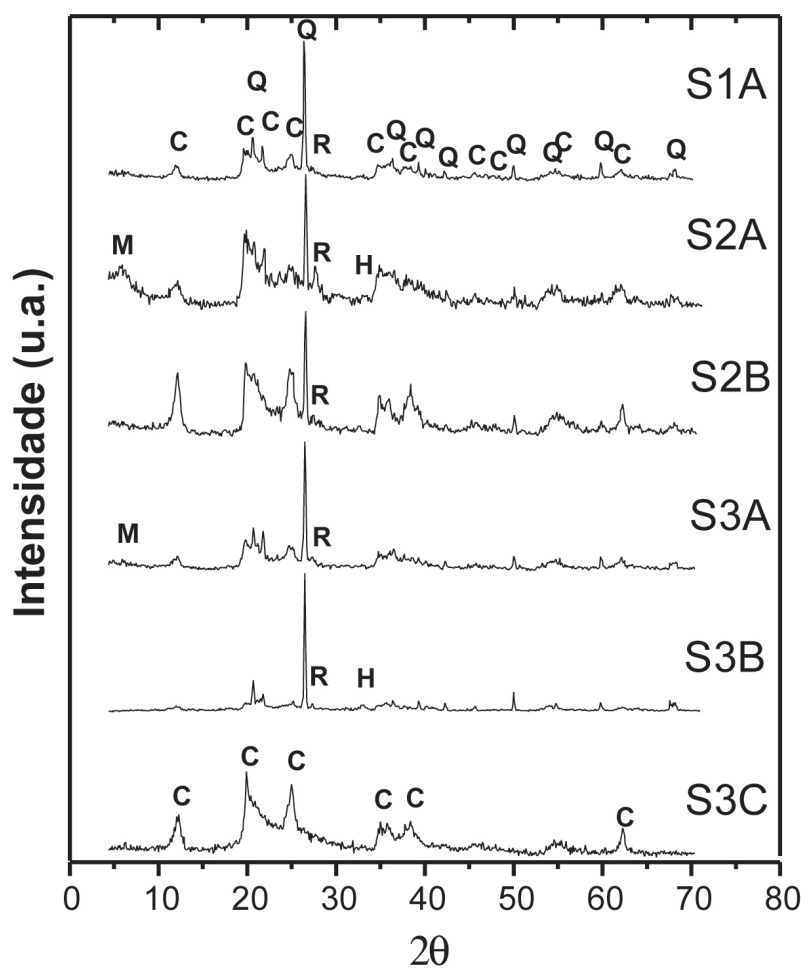

Figura 1: Difratogramas de raios $\mathrm{X}$ das amostras de solos. Alguns picos que correspondem a cada fase cristalina são indicados como: C (caulinita), Q (quartzo), M (argilomineral do tipo 2:1), H (óxido ou hidróxido de ferro) e R (rutilo).

[Figure 1: X-ray diffraction patterns of the soil samples. Peaks corresponding to each crystalline phase are indicated as: $C$ (kaolinite), $Q$ (quartz), $M$ (2:1 clay minerals), $H$ (iron oxide or hydroxide) and $R$ (rutile).]

Tabela II - Composição química das amostras de solos (\% em peso)

[Table II - Chemical composition of the soil samples (wt. \%).]

\begin{tabular}{llllllllllll}
\hline Amostra & $\mathrm{SiO}_{2}$ & $\mathrm{Al}_{2} \mathrm{O}_{3}$ & $\mathrm{Fe}_{2} \mathrm{O}_{3}$ & $\mathrm{MnO}_{2}$ & $\mathrm{Na}_{2} \mathrm{O}$ & $\mathrm{MgO}$ & $\mathrm{K}_{2} \mathrm{O}$ & $\mathrm{CaO}$ & $\mathrm{ZnO}$ & $\mathrm{CuO}$ & $\mathrm{PF}$ \\
\hline S1A & 62,23 & 16,32 & 5,15 & 0,05 & 0,11 & 0,21 & 0,18 & 0,04 & 0,01 & 0,01 & 13,29 \\
S2A & 60,25 & 8,9 & 11,20 & 0,23 & 0,11 & 0,22 & 0,11 & 0,04 & 0,01 & 0,01 & 16,56 \\
S2B & 54,23 & 19,12 & 4,50 & 0,04 & 0,10 & 0,14 & 0,14 & 0,05 & 0,01 & 0,03 & 19,01 \\
S3A & 58,03 & 15,89 & 7,54 & 0,37 & 0,10 & 0,21 & 0,22 & 0,04 & 0,01 & 0,02 & 14,70 \\
S3B & 63,33 & 8,59 & 11,50 & 0,23 & 0,03 & 0,13 & 0,08 & 0,01 & 0,01 & 0,01 & 14,04 \\
S3C & 55,10 & 14,44 & 5,50 & 0,05 & 0,02 & 0,13 & 0,06 & 0,04 & 0,01 & 0,01 & 24,32 \\
\hline
\end{tabular}


presentes, em menor quantidade ou menos cristalizadas, como evidencia a má definição e o alargamento dos picos de difração de menor intensidade.

O mineral de argila predominante em todas as amostras é a caulinita. Observa-se, também, a presença do mineral rutilo. Nos difratogramas de algumas amostras podem ser detectados picos de baixa intensidade que poderiam ser atribuídos à presença de argilominerais do tipo 2:1 e de óxido ou hidróxido de ferro, hematita ou goetita, por exemplo.

Os espectros Mössbauer de ${ }^{57} \mathrm{Fe}$ das amostras, medidos na temperatura ambiente, são apresentados na Fig. 2. Os espectros são dubletos simétricos característicos de átomos $\mathrm{Fe}$ experimentando uma interação nuclear do tipo quadrupolar elétrica. Os ajustes destes espectros foram efetuados utilizando-se um único dubleto. Os resultados dos ajustes indicam valores do desdobramento quadrupolar, $\mathrm{EQ}$, que variam entre $0,54 \mathrm{~mm} / \mathrm{s}$ e $0,59 \mathrm{~mm} / \mathrm{s}$, e do deslocamento isomérico, IS, relativo ao $\alpha-\mathrm{Fe}$, que variam entre $0,36 \mathrm{~mm} / \mathrm{s} \mathrm{e}$ $0,38 \mathrm{~mm} / \mathrm{s}$. Estes valores estão bem próximos dos valores característicos da presença de $\mathrm{Fe}^{3+}$ como substituto de $\mathrm{Al}$ nos sítios octaédricos do mineral de argila caulinita; segundo [6], $\mathrm{EQ}=0,57 \mathrm{~mm} / \mathrm{s}$ e IS $=0,35 \mathrm{~mm} / \mathrm{s}$ e segundo [7], $\mathrm{EQ}=0,58$

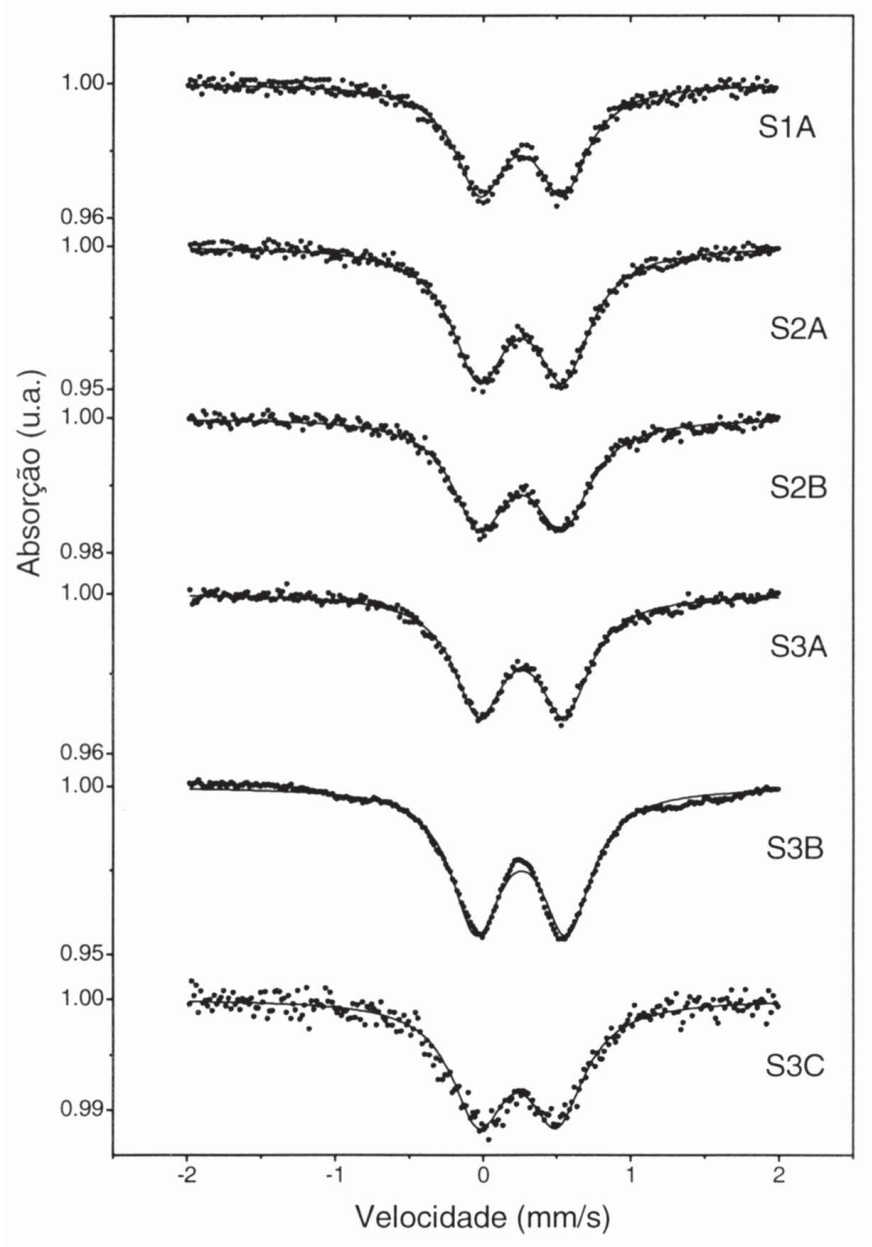

Figura 2: Espectros Mössbauer à temperatura ambiente das amostras de solos.

[Figure 2: Room temperature Mössbauer spectra of the soil samples.] $\mathrm{mm} / \mathrm{s}$ e IS $=0,37 \mathrm{~mm} / \mathrm{s}$. É bem conhecido que o Fe constitui uma das principais impurezas que podem ser encontradas nos minerais de argila, principalmente na caulinita, onde pode substituir, em grande quantidade, os átomos de Al que ocupam os sítios octaédricos da estrutura e, ocasionalmente os átomos de $\mathrm{Si}$ que ocupam os sítios tetraédricos. Também é conhecido que a incorporação de Fe na estrutura da caulinita apresenta

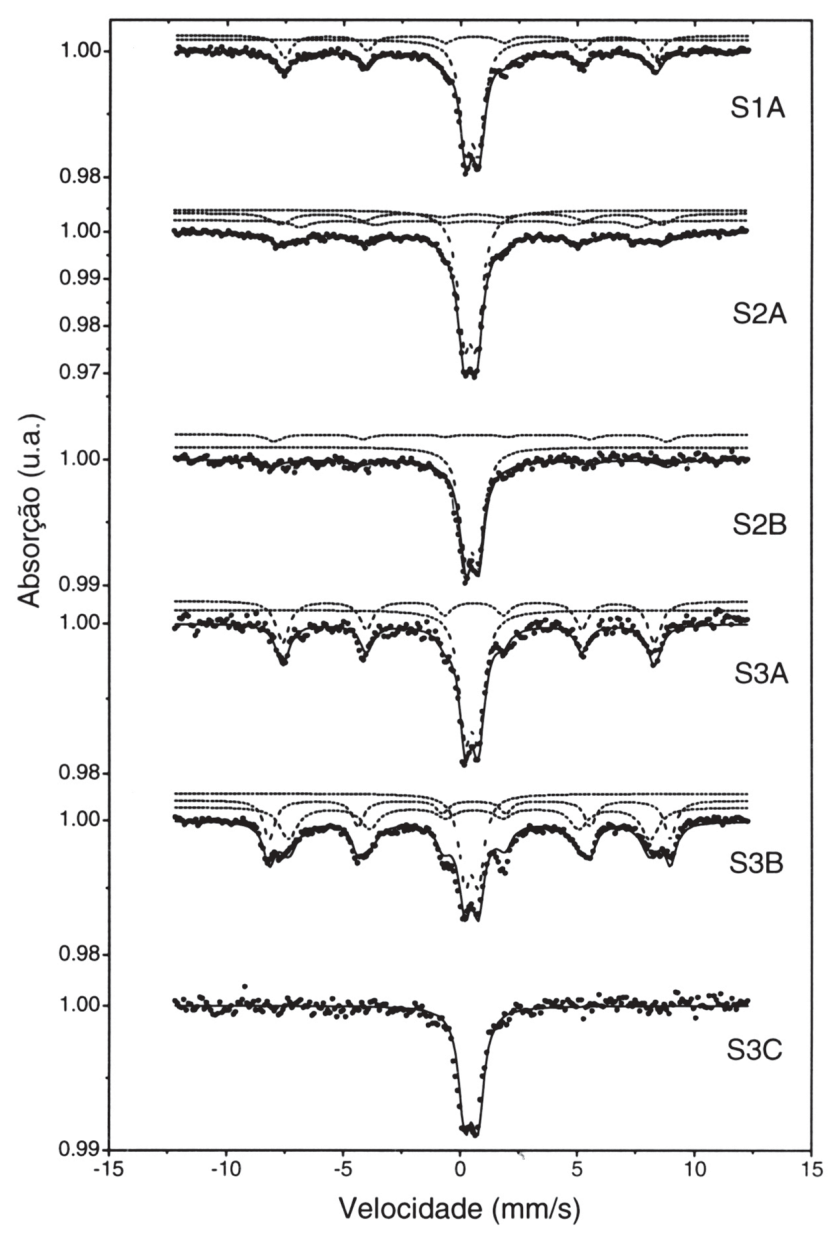

Figura 3: Espectros Mössbauer à temperatura de $77 \mathrm{~K}$ das amostras de solos.

[Figure 3: Mössbauer spectra of the soil samples at $77 \mathrm{~K}$.]

Tabela III - Parâmetros hiperfinos do espectro Mössbauer a $77 \mathrm{~K}$ para a amostra S1A.

[Table III - Hyperfine parameters of the Mössbauer spectrum at $77 \mathrm{~K}$ for the S1A sample.]

\begin{tabular}{|c|c|c|c|c|c|c|}
\hline S1A & $\begin{array}{c}\mathrm{IS} \\
(\mathrm{mms} /)\end{array}$ & $\begin{array}{c}\text { EQ } \\
(\mathrm{mm} / \mathrm{s})\end{array}$ & $\begin{array}{l}\text { BHF } \\
(\mathrm{T})\end{array}$ & $\begin{array}{c}\text { Wid } \\
(\mathrm{mm} / \mathrm{s})\end{array}$ & $\begin{array}{r}\text { Area } \\
(\%)\end{array}$ & Fases \\
\hline & $\pm 0,05$ & $\pm 0,05$ & $\pm 0,05$ & $\pm 0,05$ & \pm 1 & \\
\hline 1 & 0,49 & $-0,21$ & 49,0 & 0,66 & 34 & $\begin{array}{c}\alpha \text {-FEOOH } \\
\text { Goetita }\end{array}$ \\
\hline 2 & 0,48 & 0,55 & - & 0,60 & 66 & Caulinita \\
\hline
\end{tabular}


Tabela IV - Parâmetros hiperfinos dos espectros Mössbauer a $77 \mathrm{~K}$ para as amostras S2A e S2B. [Table IV - Hyperfine parameters of the Mössbauer spectra at $77 \mathrm{~K}$ for the samples S2A and S2B.]

\begin{tabular}{ccccccc}
\hline $2 \mathrm{~A}$ & $\begin{array}{c}\mathrm{IS} \\
(\mathrm{mms} /)\end{array}$ & $\begin{array}{c}\mathrm{EQ} \\
(\mathrm{mm} / \mathrm{s})\end{array}$ & $\begin{array}{c}\text { BHF } \\
(\mathrm{T})\end{array}$ & $\begin{array}{c}\text { Wid } \\
(\mathrm{mm} / \mathrm{s})\end{array}$ & $\begin{array}{c}\text { Área } \\
(\%)\end{array}$ & Fases \\
& $\pm 0,05$ & $\pm 0,05$ & $\pm 0,5$ & $\pm 0,05$ & \pm 1 & \\
\hline 1 & 0,44 & $-0,26$ & 46,0 & 1,30 & 21 & $\alpha$-FeOOH Goetita \\
2 & 0,49 & $-0,13$ & 50,8 & 0,70 & 12 & $\alpha-\mathrm{Fe} \neq_{2} \mathrm{O}_{3}$ Hematita \\
3 & 0,49 & 0,54 & - & 0,68 & 67 & Caulinita \\
\hline $2 \mathrm{~B}$ & $\mathrm{IS}$ & $\mathrm{EQ}$ & $\mathrm{BHF}$ & Wid & Área & Fases \\
& $(\mathrm{mms} /)$ & $(\mathrm{mm} / \mathrm{s})$ & $(\mathrm{T})$ & $(\mathrm{mm} / \mathrm{s})$ & $(\%)$ & \\
& $\pm 0,05$ & $\pm 0,05$ & $\pm 0,5$ & $\pm 0,05$ & \pm 1 & \\
\hline 1 & 0,50 & $-0,26$ & 49,0 & 0,90 & 17 & $\alpha-F e O O H$ Goetita \\
2 & 0,49 & 0,55 & - & 0,63 & 83 & Caulinita \\
\hline
\end{tabular}

Tabela V- Parâmetros hiperfinos dos espectros Mössbauer a 77K para as amostras S3A, S3B e S3C. [Table V-Hyperfine parameters of the Mössbauer spectra at $77 \mathrm{~K}$ for the samples S3A, S3B and S3C.]

\begin{tabular}{|c|c|c|c|c|c|c|}
\hline $3 \mathrm{~A}$ & $\begin{array}{c}\text { IS } \\
(\mathrm{mms} /)\end{array}$ & $\begin{array}{c}\text { Deq } \\
(\mathrm{mm} / \mathrm{s})\end{array}$ & $\begin{array}{l}\text { BHF } \\
(\mathrm{T})\end{array}$ & $\begin{array}{c}\text { Wid } \\
(\mathrm{mm} / \mathrm{s})\end{array}$ & $\begin{array}{c}\text { Área } \\
(\%)\end{array}$ & Fases \\
\hline & $\pm 0,05$ & $\pm 0,05$ & $\pm 0,5$ & $\pm 0,05$ & \pm 1 & \\
\hline 1 & 0,49 & $-0,24$ & 49,0 & 0,70 & 45 & $\alpha$-FEOOHGoetita \\
\hline 2 & 0,49 & 0,56 & - & 0,60 & 55 & Caulinita \\
\hline \multirow[t]{2}{*}{$3 \mathrm{~B}$} & $\begin{array}{c}\mathrm{IS} \\
(\mathrm{mms} /)\end{array}$ & $\begin{array}{c}\text { Deq } \\
(\mathrm{mm} / \mathrm{s})\end{array}$ & $\begin{array}{l}\text { BHF } \\
(\mathrm{T})\end{array}$ & $\begin{array}{c}\text { Wid } \\
(\mathrm{mm} / \mathrm{s})\end{array}$ & $\begin{array}{c}\text { Área } \\
(\%)\end{array}$ & Fases \\
\hline & $\pm 0,05$ & $\pm 0,05$ & $\pm 0,5$ & $\pm 0,05$ & \pm 1 & \\
\hline 1 & 0,49 & $-0,22$ & 49,2 & 1,00 & 58 & $\alpha$-FEOOH Goetita \\
\hline 2 & 0,50 & $-0,16$ & 53,6 & 0,70 & 15,0 & $\alpha-\mathrm{Fe}_{2} \mathrm{O}_{3}$ Hematita \\
\hline 3 & 0,49 & 0,58 & - & 0,60 & 27 & Caulinita \\
\hline \multirow[t]{2}{*}{$3 \mathrm{C}$} & $\begin{array}{c}\mathrm{IS} \\
(\mathrm{mms} /)\end{array}$ & $\begin{array}{c}\text { Deq } \\
(\mathrm{mm} / \mathrm{s})\end{array}$ & $\begin{array}{l}\text { BHF } \\
\text { (T) }\end{array}$ & $\begin{array}{c}\text { Wid } \\
(\mathrm{mm} / \mathrm{s})\end{array}$ & $\begin{array}{c}\text { Área } \\
(\%)\end{array}$ & Fases \\
\hline & $\pm 0,05$ & $\pm 0,05$ & $\pm 0,5$ & $\pm 0,05$ & \pm 1 & \\
\hline 1 & 0,45 & 0,55 & - & 0,70 & - & Caulinita \\
\hline
\end{tabular}

uma acentuada correlação inversa com o grau de cristalinidade do mineral.

À temperatura de $77 \mathrm{~K}$, Fig. 3, todas as amostras, exceto a S3C, apresentam um espectro Mössbauer composto pela superposição de um dubleto simétrico com uma componente magnética alargada (sub-espectro padrão de seis linhas característico de átomos de Fe experimentando uma interação magnética) cujo grau de definição varia de uma amostra para outra. Estes espectros foram ajustados utilizando um dubleto e um ou dois sextetos considerando-se o desdobramento 
hiperfino magnético. O espectro da amostra S3C foi ajustado utilizando um único dubleto. Os dados obtidos dos ajustes são apresentados nas Tabelas III, IV e V. Como pode ser observado, o dubleto em todos os espectros apresenta valores do EQ similares aos obtidos à temperatura ambiente para a respectiva amostra, confirmando a presença do mineral caulinita em todas elas. $\mathrm{O}$ ajuste da parte magnética revela um campo magnético hiperfino, BHF, em torno de $49 \mathrm{~T}$ em todas as amostras. Este valor é similar ao valor típico relatado na literatura para o mineral goetita $[6,8]$.

A necessidade de ajustar os espectros Mössbauer a $77 \mathrm{~K}$ das amostras S2A e S3B utilizando-se um segundo sexteto revela a presença de um BHF adicional de 50 e $55 \mathrm{~T}$ nestas amostras, respectivamente. Estes valores de BHF são próximos do valor característico do mineral hematita [6-9]. O minerais de Fe goetita $(\alpha-\mathrm{FeOOH})$ e hematita $\left(\alpha-\mathrm{Fe}_{2} \mathrm{O}_{3}\right)$ são compostos comuns nos solos de cor amarelada ou vermelha e em solos cauliníticos. Em geral, são encontrados na forma magnética à temperatura ambiente apresentando um espectro Mössbauer de seis linhas (sexteto). Mas, quando o tamanho das partículas destes minerais é menor do que $100 \AA ̊$ obtemos à temperatura ambiente no espectro Mössbauer um dubleto resultante do colapso do sexteto [4]. Este fenômeno é associado a uma relaxação superparamagnética que ocorre quando a direção de magnetização das partículas flutua entre as varias direções de fácil magnetização e quando o tempo de relaxação é curto comparado com a escala de tempo da medida.

Os resultados da análise via espectroscopia Mössbauer confirmam a presença, em todas as amostras, do mineral caulinita com baixa cristalinidade. Também parecem indicar a presença do mineral goetita em todas as amostras, exceto a $\mathrm{S} 3 \mathrm{C}$, além de hematita nas amostras S2A e S3B. Ambos os minerais, goetita e hematita estariam dispersos na forma de finos precipitados com distribuição de tamanho de partículas.

A Tabela VI mostra os limites de Atterberg das amostras dos solos estudados. Uma vez que o limite de plasticidade das amostras varia de 24,5 a $43,8 \%$ é possível considerá-los materiais com alta plasticidade (L.P. $>15 \%,[10])$. O limite de liquidez da maioria das amostras (exceto a amostra S1A) é superior a $50 \%$, o que indica que estes solos apresentam uma alta compressibilidade. De acordo com [11], no caso específico da tecnologia cerâmica para moldagem de tijolos furados por extrusão, as faixas de plasticidade recomendadas para argilas plásticas cauliníticas contendo matéria orgânica correspondem a: L.P. variando de $15,42 \%$ a $36,72 \%$, L.L. variando de $24,70 \%$ a $71,00 \%$ e I.P. variando de $6,82 \%$ a $34,28 \%$. Cinco das amostras estudadas (S1A, S2A, S2B, S3A e S3B) apresentam índices de plasticidade dentro das faixas recomendadas. A amostra do solo S3C apresenta um L.P. acima de $36,72 \%$ fato que pode estar associado à presença de uma grande quantidade de matéria orgânica, como sugere a cor do solo, preta, e a relativamente alta porcentagem de perda ao fogo (Tabela II).

A análise granulométrica demonstrou que todas as amostras apresentam uma porcentagem em peso de partículas com diâmetro superior a $0,074 \mathrm{~mm}$, que é inferior a 18,5\%. Assim sendo, os solos estudados podem ser classificados como solos finos. Os dados da distribuição de tamanho de partículas das amostras são apresentados na Tabela VI. A concentração de partículas com diâmetro menor que $2 \mu \mathrm{m}$ varia de 18 até $42 \%$, entre $2 \mu \mathrm{m}$ e $20 \mu \mathrm{m}$ varia de 32 até $46 \%$, e com diâmetro maior que $20 \mu \mathrm{m}$ varia de 25 até $36 \%$.

Em geral, uma massa cerâmica não pode ser constituída somente de argilas plásticas, porque apresenta grandes dificuldades no processamento, desde a conformação das peças, incluindo ainda a secagem e a queima. A solução destes problemas é fazer uma composição granulométrica adequada, dosando-se grãos finos, médios e grossos. Neste caso, o diagrama de Winkler é a ferramenta ideal para se fazer este estudo.

Comparando composição granulométrica das amostras de solos estudados com o diagrama de Winkler [12], Tabela VII, pode ser observado que a distribuição de tamanho de partículas dos solos S1A e S3A corresponde com aquela adequada para a fabricação de produtos de qualidade. A do solo S2A enquadra-se na faixa utilizada na fabricação de telhas e a dos solos S2B e S3B, na utilizada para fabricar tijolos furados. A distribuição granulométrica do solo $\mathrm{S} 3 \mathrm{C}$ é a conveniente para a produção de tijolos maciços.

Tabela VI - Limites de Atterberg e distribuição de tamanho de partículas nos solos. [Table VI-Atterberg limits and sizes distribution of particles in the soils.]

\begin{tabular}{clllccc}
\hline & \multicolumn{2}{c}{ Limites de Atterberg (\%) } & \multicolumn{4}{c}{ Composição granulométrica (\%) } \\
\hline Amostra & L.L. & L.P. & I.P. & $<2 \mu \mathrm{m}$ & $2-20 \mu \mathrm{m}$ & $>20 \mu \mathrm{m}$ \\
\hline S1A & 44,8 & 24,5 & 20,3 & 40 & 32 & 28 \\
S1B & 53,2 & 35,7 & 17,5 & 58 & 20 & 22 \\
S2A & 51,6 & 34,6 & 17,0 & 34 & 34 & 32 \\
S2B & 50,2 & 25,2 & 25,0 & 29 & 43 & 28 \\
S3A & 50,8 & 29,9 & 20,9 & 42 & 33 & 25 \\
S3B & 52,5 & 25,4 & 27,1 & 28 & 40 & 32 \\
S3C & 57,8 & 43,8 & 13,7 & 18 & 46 & 36 \\
\hline
\end{tabular}

L.L.= limite de liquidez; L.P.= limite de plasticidade; I.P.= indice de plasticidade 
Tabela VII - Composição granulométrica dos produtos da cerâmica vermelha de acordo com o diagrama de Winkler [8].

[Table VII - Granulometric composition of products of the red ceramic according to the Winkler diagram [8].]

\begin{tabular}{llll}
\hline Regiões & \multicolumn{3}{l}{ Composição granulométrica } \\
\hline Tipos de Produtos & $<2 \mu \mathrm{m}$ & 2 a $20 \mu \mathrm{m}$ & $>2 \mu \mathrm{m}$ \\
\hline Materiais de qualidade com dificuldade de produção & 40 a 50 & 20 a 40 & 20 a 30 \\
Telhas, capas & 30 a 40 & 20 a 50 & 20 a 40 \\
Tijolos furados & 20 a 30 & 20 a 55 & 20 a 50 \\
Tijolos maciços & 15 a 20 & 20 a 55 & 25 a 55 \\
\hline
\end{tabular}

\section{CONCLUSÕES}

1 - A análise por meio de difração de raios X mostra que o argilomineral predominante em todas as amostras de solo é a caulinita, e indica ainda a presença de quartzo e rutilo. Sugere também a presença de argilominerais do tipo 2:1 e de óxido ou hidróxido de ferro, em algumas amostras.

2 - O estudo por espectroscopia Mössbauer confirma a presença do argilomineral caulinita com baixa cristalinidade. Indica também a presença do mineral goetita em todos as amostras, exceto a $\mathrm{S} 3 \mathrm{C}$, e a do mineral hematita nas amostras S2A e S3B, ambos na forma de partículas ultrafinas (nanopartículas).

3- Os testes físicos efetuados nas amostras indicam que os solos são finos e apresentam características granulométricas e de plasticidade que podem ser aproveitadas para a produção de materiais cerâmicos para construção ou objetos ornamentais.

\section{AGRADECIMENTOS}

Os autores agradecem ao CNPq pelo financiamento parcial recebido e à FUNAI, Passo Fundo, pelas amostras de solos e pela oportunidade de estabelecer-se uma colaboração.

\section{REFERÊNCIAS}

[1] J. K. Mitchell, Fundamentals of soil behavior. John Wiley \& Sons, Inc., $2^{\text {nd }}$ Ed., New York (1992) p.437.

[2] C. M. F. Viera, J. N. F. de Holanda, D. G. Pinatti, Cerâmica 46, 297 (2000) 14.

[3] A. P. F. Albers, F. G. Melchiades, R. Machado, J. B. Baldo, A. O. Boschi, Cerâmica 48, 305 (2002) 34.

[4] N. N. Greenwood, T. C. Gibb, Mössbauer Spectroscopy. Chapman and Hall Ltd., London (1971) p. 659.

[5] E. Murad, Hyperfine Interactions 117 (1998) 39.

[6] O. Castelein, L. Aldon, J. Olivier-Fourcade, J. C. Jumas, J. P. Bonnet, P. Blanchart, J. Eur. Ceram. Soc. 22 (2002) 1767.

[7] S. Petit, A. Decarreau, Clay Miner. 25 (1990) 181.

[8] J. Silver, M. Sweeney, I. E. G. Morrison, Thermochim. Acta 35 (1980) 153.

[9] R. A. Borzi, S. J. Stewart, G. Punte, R. C. Mercader, M. Vasquez-Mansilla, R. D. Zysler, E. D. Cabanillas, J. Magn. Magn. Mater. 205 (1999) 234.

[10] S. R. Teixeira, S. A. Souza, C. A. I. Moura, Cerâmica 47, 304 (2001) 204.

[11] L. F. A. Campos, R. S. de Macedo, P. K. Kiyohara, H. C. Ferreira, Cerâmica 45, 295 (1999) 140.

[12] S. Pracidelli, G. F. Melchiades, Cerâmica Industrial 2, 01/02 (1997) 31.

(Rec. 21/08/03, Rev. 30/10/03, Ac. 20/02/04) 